\title{
GaAs metal-semiconductor field effect transistor with InGaP/GaAs multiquantum barrier buffer layer
}

\author{
Ching-Ting Lee ${ }^{\mathrm{a}, *}$, Kuo-Chuan Shyu ${ }^{\mathrm{a}}$, Iang-Jeng Lin ${ }^{\mathrm{a}}$, Hao-Hsiung Lin ${ }^{\mathrm{b}}$ \\ ${ }^{a}$ Institute of Optical Sciences, National Central University, Chung-Li, Taiwan, ROC \\ ${ }^{\mathrm{b}}$ Department of Electrical Engineering, National Taiwan University, Taipei, Taiwan, ROC
}

\begin{abstract}
A novel metal-semiconductor field effect transistor (MESFET) with $\mathrm{InGaP} / \mathrm{GaAs}$ multiple quantum barrier (MQB) buffer layer was studied. Since the effective potential barrier height is enhanced using the $\mathrm{InGaP} / \mathrm{GaAs} \mathrm{MQB}$ structure, good performances of electronic and optical isolations are achieved. A configuration with the MESFET and sidegate electrode was fabricated to demonstrate the function of the InGaP/GaAs MQB structure. (C) 2000 Elsevier Science S.A. All rights reserved.
\end{abstract}

Keywords: Metal-semiconductor field effect transistor (MESFET); InGaP/GaAs; Multiple quantum barrier (MQB)

\section{Introduction}

Since their introduction in 1970, GaAs metal-semiconductor field effect transistors (MESFETs) have occupied an important niche in the high-speed electronic and optoelectronic industries. Monolithic microwave integrated circuits, optical transmitters and optical receivers based on GaAs MESFET have been developed in analog and digital systems. The basic advantages of GaAs MESFETs include a higher electron mobility, leading to higher speed operation, and semi-insulating GaAs substrates, which allow one to reduce the parasitic capacitances and simplify the associated fabrication process. However, the device density and performance of GaAs integrated circuits would be significantly limited by the parasitic interaction and sidegating effect among active devices. The physical basis of this effect was deduced from an increase in the space-charge layer width at the channel-substrate interface of the MESFETs [1,2]. To improve the electronic isolation and optically induced isolation influenced from GaAs substrates, various buffer layers grown between channel and substrate have been employed widely. Multiple quantum barrier (MQB) structures have demonstrated novel property and ability to in-

\footnotetext{
* Corresponding author. Tel.: + 886-3-4263914; fax: + 886-34252897.

E-mail address: t260003@cc.ncu.edu.tw (C.-T. Lee)
}

crease the effective potential barrier height theoretically and experimentally [3,4]. Recently, electronic sidegating effect improvement and optically induced sidegating effect isolation of GaAs MESFETs using AlGaAs/ GaAs MQB structure grown between channel and substrate as a buffer layer have been reported $[5,6]$. However, the resultant performances of the associated devices and integrated circuits would be degraded by the persistent photoconductivity (PPC) effect commonly found in $\mathrm{AlGaAs}$ layer due to its donor complex (DX) centers and other deep levels [7,8]. Compared with the AlGaAs material, the aluminum-free, wide bandgap $\operatorname{In}_{0.5} \mathrm{Ga}_{0.5} \mathrm{P}$ (referred to as $\mathrm{InGaP}$ ) material lattice-matched to GaAs was shown to exhibit basic advantages included lower DX centers and deep levels [9], larger band discontinuity in the $\mathrm{InGaP} / \mathrm{GaAs}$ heterojunction [10], lower surface recombination velocity at the InGaP-GaAs interface [11] and higher selective etching rate between InGaP and GaAs [12]. These advantages make $\mathrm{InGaP} / \mathrm{GaAs} \mathrm{MQB}$ structure a promising alternative for $\mathrm{AlGaAs} / \mathrm{GaAs} \mathrm{MQB}$ structure as a buffer layer in GaAs MESFETs. High performances and thermal reliability of GaAs metal-semiconductor-metal photodetectors with InGaP capping and buffer layers were reported recently [13]. In this study, we used $\mathrm{InGaP} / \mathrm{GaAs}$ MQB structure grown between channel and substrate as a buffer layer in GaAs MESFETs. The improvement of electronic and optically induced isolations was reported. 


\section{Device structure and fabrication processes}

The epitaxial layers used in these GaAs MESFETs, shown in Fig. 1, were grown on (100) semi-insulating GaAs substrates using molecular beam epitaxial system. After first growing an undoped GaAs (300-nm) buffer layer, the second InGaP/GaAs MQB buffer layer, a

\begin{tabular}{|cc|}
\hline $\mathrm{n}^{+}-$GaAs capping layer, $\mathrm{n}=5 \times 10^{18} \mathrm{~cm}^{-3}$ & $20 \mathrm{~nm}$ \\
\hline $\mathrm{n}$-GaAs channel layer, $\mathrm{n}=3 \times 10^{17} \mathrm{~cm}^{-3}$ & $300 \mathrm{~nm}$ \\
\hline InGaP/GaAs MQB layer & $64.5 \mathrm{~nm}$ \\
\hline undoped GaAs buffer layer & $300 \mathrm{~nm}$ \\
\hline \\
S.I. GaAs substrate \\
\hline
\end{tabular}

Fig. 1. Schematic epitaxial structure.

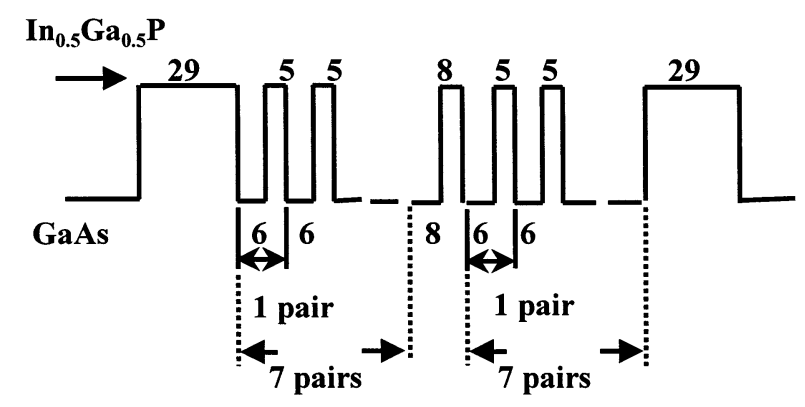

(a)

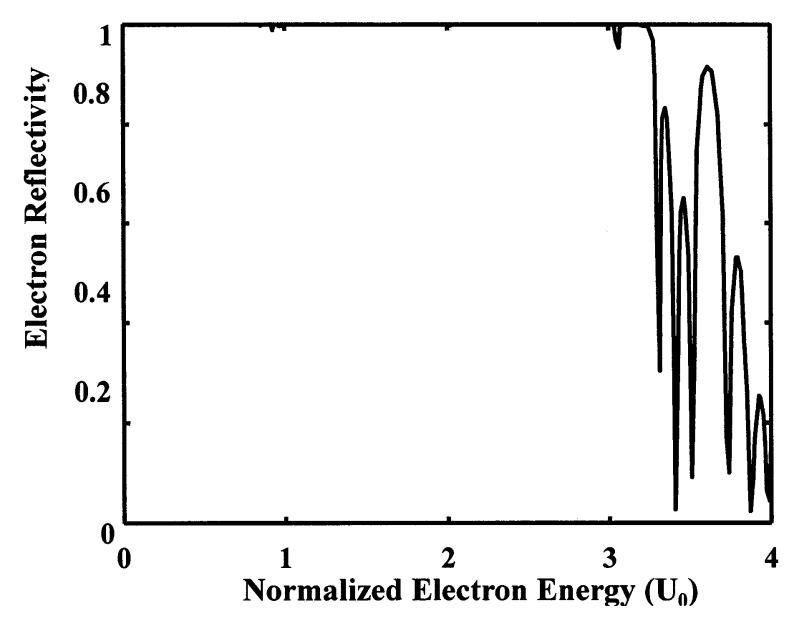

(b)

Fig. 2. (a) Schematic InGaP/GaAs multiple quantum barrier structure in monolayer and (b) the dependence of electron reflectivity on the normalized electron energy.

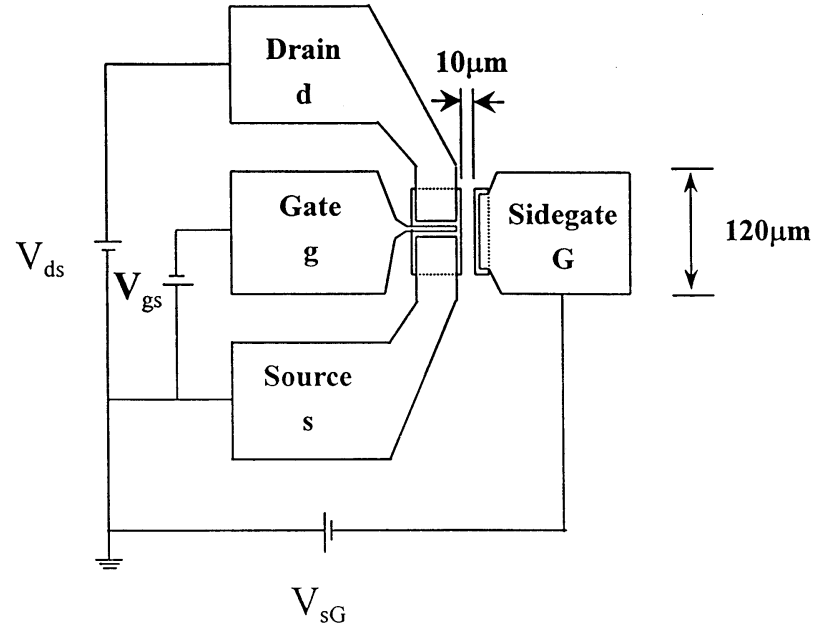

Fig. 3. Schematic top view of the MESFET test configuration.

Si-doped GaAs channel layer $\left(300 \mathrm{~nm}, 3 \times 10^{17} \mathrm{~cm}^{-3}\right)$, and a heavily Si-doped GaAs capping layer $(20 \mathrm{~nm}$, $5 \times 10^{18} \mathrm{~cm}^{-3}$ ) were grown sequentially. This heavily Si-doped GaAs capping layer was grown for good ohmic performance of source and drain. The InGaP/ GaAs MQB buffer layer structure designed with three periodic stacks is described as $\left(B_{0} ; W_{1}, B_{1}, P_{1} ; W_{2}, B_{2}, P_{2} ; W_{3}, B_{3}, P_{3} ; B_{0}\right)$, where $B_{0}$ is the number of monolayers of the InGaP barrier grown on the top and bottom regions of the MQB buffer layer structure; $W_{i}$ and $B_{i}$ are the number of monolayers of the following GaAs well and InGaP barrier, respectively; and $P_{i}$ is the pair number of the GaAs well and InGaP barrier. The designed $\mathrm{InGaP} / \mathrm{GaAs} \mathrm{MQB}$ buffer layer structure is constructed by $(29 ; 6,5,7 ; 8,8,1 ; 6,5,7 ; 29)$ as shown in Fig. 2(a). The MQB buffer layer consists of 228 monolayers. Its total thickness is $\sim 64.5 \mathrm{~nm}$. The thick InGaP barrier grown on the top and bottom regions of the MQB buffer layer, contacted with the GaAs channel layer and GaAs buffer layer, respectively, is to evade low-energy electron tunneling.

Assuming the band offset $\Delta E_{\mathrm{c}}=223 \mathrm{meV}$ for the InGaP/GaAs heterostructure, the effective mass of electron in GaAs well and InGaP barrier is 0.067 and 0.11 $m_{0}$, respectively $[14,15]$, where $m_{0}$ is the electron rest mass; the reflectivity of the electron wave was calculated using the transfer matrix method $[16,17]$. For this designed $\mathrm{InGaP} / \mathrm{GaAs} \mathrm{MQB}$ structure, the dependence of the electron reflectivity on the electron energy normalized with $U_{0}$ is shown in Fig. 2(b), where $U_{0}$ is the potential barrier height for an undoped $\mathrm{InGaP} / \mathrm{GaAs}$ heterostructure. It can be seen that the effective potential barrier height of the designed $\mathrm{InGaP} / \mathrm{GaAs} \mathrm{MQB}$ structure is estimated to increase to $3.2 U_{0}$.

Standard photolithography and lift-off techniques were used to fabricate MESFET and sidegate electrode. Fig. 3 shows the schematic top view of the devices. The MESFET mesa $\left(25 \times 60 \mu \mathrm{m}^{2}\right)$ and sidegate mesa $(10 \times$ 
$60 \mu \mathrm{m}^{2}$ ) separated with a width of $10 \mu \mathrm{m}$ were obtained by etching through the $\mathrm{n}^{+}$-GaAS capping layer down to the undoped GaAs buffer layer using $\mathrm{Si}_{3} \mathrm{~N}_{4}$ mask. During the mesa etching process, the GaAs layers and

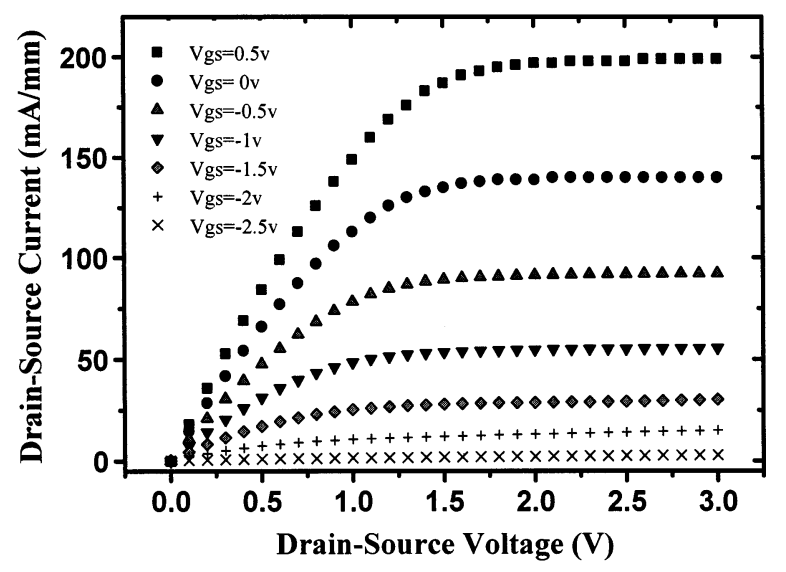

Fig. 4. Typical drain-source current $\left(I_{\mathrm{ds}}\right)$ as a function of drain-source voltage $\left(V_{\mathrm{ds}}\right)$ for various gate-source voltages $\left(V_{\mathrm{gs}}\right)$.

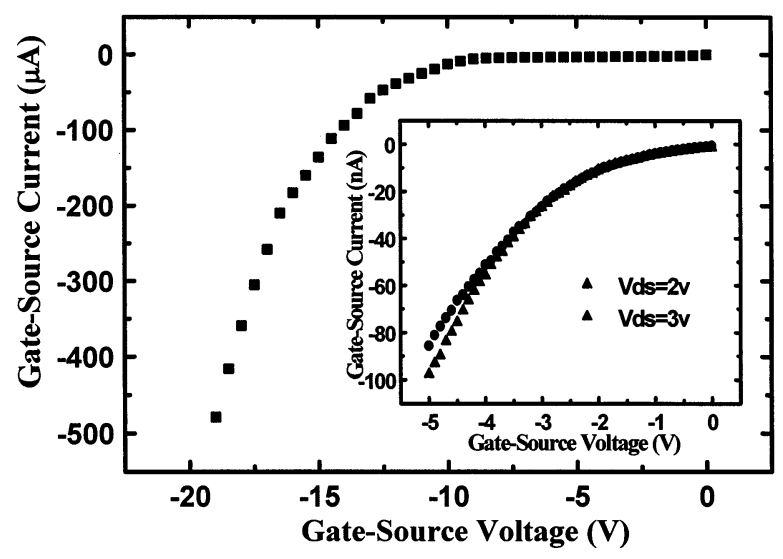

Fig. 5. Typical gate-source current $\left(I_{\mathrm{gs}}\right)$ as a function of drain-source voltage $\left(V_{\mathrm{gs}}\right)$ for floating drain and drain-source voltage $V_{\mathrm{ds}}=2$ and $3 \mathrm{~V}$.

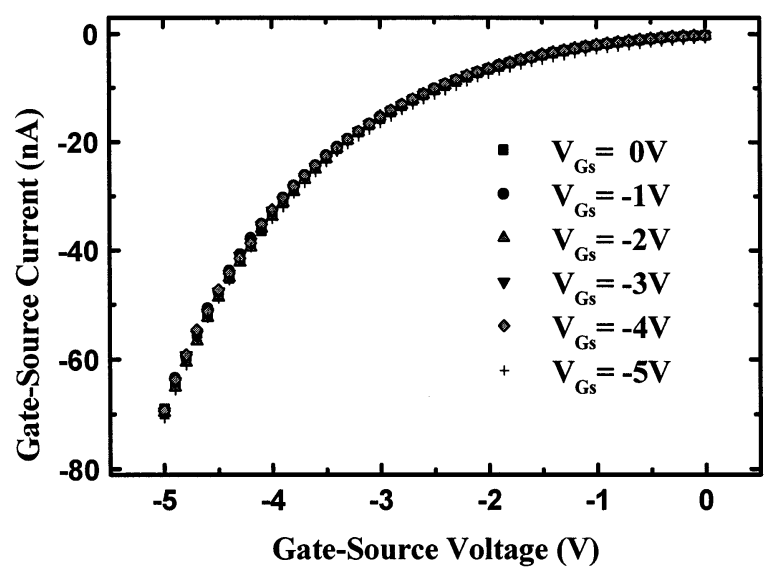

Fig. 6. Typical gate-source current $\left(I_{\mathrm{gs}}\right)$ as a function of gate-source voltage $\left(V_{\mathrm{gs}}\right)$ for various sidegate-source voltages $\left(V_{\mathrm{GS}}\right)$.
MQB buffer layer were etched using chemical solution of $\mathrm{H}_{2} \mathrm{SO}_{4}: \mathrm{H}_{2} \mathrm{O}_{2}: \mathrm{H}_{2} \mathrm{O}(1: 10: 200)$ and $\mathrm{HCl}: \mathrm{HNO}_{3}: \mathrm{H}_{2} \mathrm{O}$ (1:1:1), respectively. After the MQB buffer layer was etched, the undoped GaAs buffer layer was then etched $\sim 50 \mathrm{~nm}$. The patterns of the source and drain regions for MESFETs and sidegate region were defined using photolithography technique. The spacing of the source and drain regions was $5 \mu \mathrm{m}$. Prior to ohmic metal deposition, the native oxidation on the surface was removed using chemical solution of $\mathrm{NH}_{4} \mathrm{OH}: \mathrm{H}_{2} \mathrm{O}$ (1:10). The ohmic contact regions of source, drain and sidegate were then formed by evaporated $\mathrm{NiGeAu} / \mathrm{Au}$ $(100 / 300 \mathrm{~nm})$ and thermally alloyed at $400^{\circ} \mathrm{C}$ for $3 \mathrm{~min}$. Following the gate recess etching down to the GaAs channel layer, a gate pattern with a length of $1 \mu \mathrm{m}$ and width of $25 \mu \mathrm{m}$ was aligned and gate metals of $\mathrm{Ti} / \mathrm{Pt} /$ $\mathrm{Au}(50 / 100 / 300 \mathrm{~nm})$ were deposited.

\section{Experimental results and discussion}

Fig. 3 shows the test structure and set-up for the MESFET isolation measurements. The measurements were carried out using an HP4145B semiconductor parameter analyzer. The d.c. characteristic of the drainsource current $\left(I_{\mathrm{ds}}\right)$ as a function of the drain-source voltage $\left(V_{\mathrm{ds}}\right)$ for the MESFET itself is shown in Fig. 4. It can be seen that this MESFET can be completely pinched off at gate-source voltage $\left(V_{\mathrm{gs}}\right)$ of $-2.5 \mathrm{~V}$. To investigate the gate performance, when the drain is floating, the measured $I_{\mathrm{gs}}$ as a function of $V_{\mathrm{gs}}$ is shown in Fig. 5. We define the breakdown voltage as the voltage when its corresponding $I_{\mathrm{gs}}$ increases rapidly. A breakdown voltage of $12.5 \mathrm{~V}$ was found. When the drain-source voltage $\left(V_{\mathrm{ds}}\right)$ was applied, the dependence of $I_{\mathrm{gs}}$ on $V_{\mathrm{gs}}$ for $V_{\mathrm{ds}}=2$ and $3 \mathrm{~V}$ as shown in Fig. 5. We can find that $I_{\mathrm{gs}}$ increases gradually with $V_{\mathrm{ds}}$ for $V_{\mathrm{gs}}$ larger than $3.6 \mathrm{~V}$.

To study the isolation function of the $\mathrm{InGaP} / \mathrm{GaAs}$ MQB buffer layer, the dependence of $I_{\mathrm{gs}}$ on $V_{\mathrm{gs}}$ for various sidegate-source voltages $\left(V_{\mathrm{GS}}\right)$ is shown in Fig. 6. It can be seen that $I_{\mathrm{gs}}$ is not influenced by $V_{\mathrm{GS}}$ for a fixed $V_{\mathrm{gs}}$ value. Therefore, using the $\mathrm{InGaP} / \mathrm{GaAs}$ MQB buffer layer structure, the electronic parasitic interaction of the MESFETs can be reduced. Furthermore, to study the photoexcited sidegating current, three kinds of laser beam were illuminated on the fabricated devices. In general, when an excitation light is illuminated on a multiple quantum barrier structure, photoexcited carriers are generated [18]. Fig. 7 shows the wavelength dependence of the sidegating current of the MESFET at light power of $0.2 \mathrm{~mW}$ and $V_{\mathrm{ds}}=2 \mathrm{~V}$. For the laser beam with a wavelength of $1300 \mathrm{~nm}$, photoexcited carriers are not generated within the $\mathrm{GaAs}$ and InGaP layers, while the crystal temperature is increased and traps are excited. Because the traps 


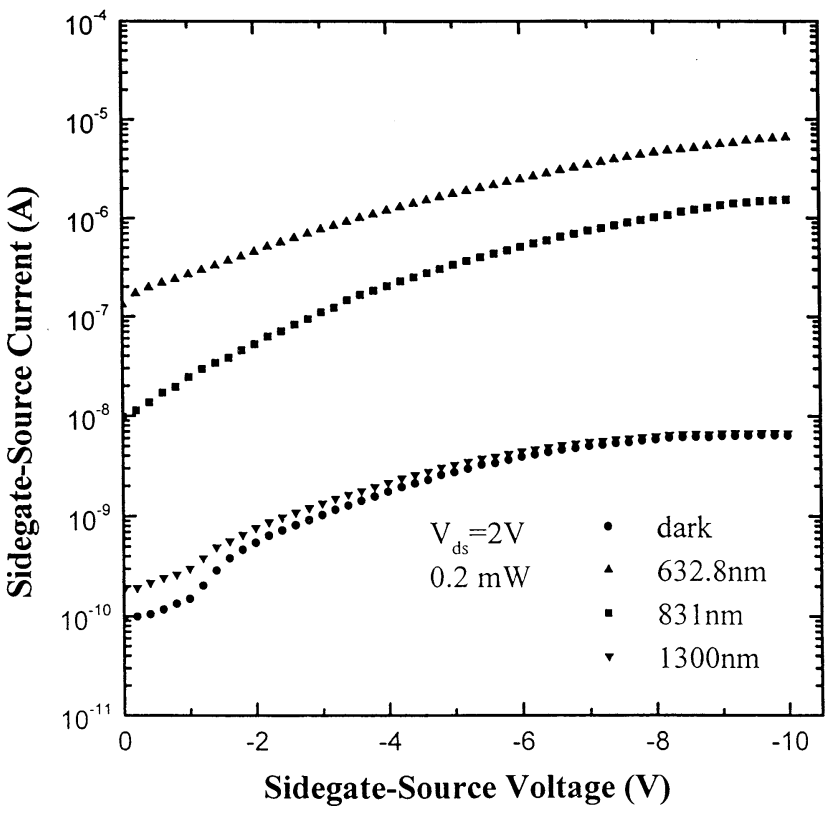

Fig. 7. The dependence of sidegate-source current $\left(I_{\mathrm{GS}}\right)$ on the sidegate-source voltage $\left(V_{\mathrm{GS}}\right)$ with $V_{\mathrm{ds}}=2 \mathrm{~V}$ and incident laser power of $0.2 \mathrm{~mW}$ of varying wavelength.

existing within the $\mathrm{InGaP} / \mathrm{GaAs} \mathrm{MQB}$ structure are very small and the band discontinuity in the $\mathrm{InGaP} /$ GaAs heterojunction is very large, the sidegate-source current for illuminating with 1300 -nm light is almost the same as the dark sidegate-source current as shown in Fig. 7. For the laser beam with a wavelength of 831 $\mathrm{nm}$, the photoexcited carriers are generated in the GaAs layers, while the photoexcited carriers are produced in both the GaAs and InGaP layers for illuminating with 632.8-nm laser beam. Therefore, the sidegate-source current for illuminating with $632.8-\mathrm{nm}$ light is larger than that of 831-nm light. According to these experimental results, the enhanced potential barrier height of the InGaP/GaAs buffer layer can result in good isolation and sidegating current suppression of the carriers.

\section{Conclusions}

A novel MESFET with InGaP/GaAs multiple quantum barrier buffer layer was reported in this paper. For the designed InGaP/GaAs $(29 ; 6,5,7 ; 8,8,1 ; 6,5,7 ; 29)$ MQB structure, the effective potential barrier height is enhanced to $3.2 U_{0}$, where $U_{0}$ is the potential barrier height of the InGaP-GaAs heterostructure. A configuration with this kind of MESFET and sidegate electrode was fabricated to investigate the function of the $\mathrm{InGaP} / \mathrm{GaAs} \mathrm{MQB}$ structure. Based on the experimen- tal results reported here, an electronic parasitic interaction reduction and optically induced effect suppression were demonstrated. According to the isolation function of the $\mathrm{InGaP} / \mathrm{GaAs} \mathrm{MQB}$ structure, high device density electronic integrated circuits and electrooptical integrated circuits can be expected using suitably designed MQB buffer layer structure. Although the performances of the MESFETs with AlGaAs/GaAs MQB buffer layer were reported previously [5,6], the InGaP/ GaAs MQB structure would be a better candidate due to the superior property of InGaP material compared with AlGaAs material.

\section{Acknowledgements}

The authors wish to acknowledge the National Science Council of the Republic of China for financial support under grant NSC 89-2215-E008-030.

\section{References}

[1] C. Kocot, C.A. Stolte, IEEE Trans. Electron. Devices 29 (1982) 1059.

[2] M.F. Chang, C.P. Lee, L.D. Hou, R.P. Vahrenkamp, C.G. Kirkpatrick, Appl. Phys. Lett. 44 (1984) 869.

[3] T. Takagi, F. Koyama, K. Iga, Jpn. J. Appl. Phys. 29 (1990) 1977.

[4] S.T. Yen, C.M. Tsai, C.P. Lee, D.C. Liu, Appl. Phys. Lett. 64 (1994) 1108.

[5] C.T. Lee, C.D. Tsai, C.Y. Wang, H.P. Shiao, T.E. Nee, J.N. Shen, Appl. Phys. Lett. 67 (1995) 2046.

[6] C.T. Lee, IEEE Trans. Electron. Devices 45 (1998) 2083.

[7] D.V. Lang, R.A. Logan, M. Jaros, Phys. Rev. B19 (1979) 1015.

[8] J.C. Liou, K.M. Lau, IEEE Trans. Electron. Devices 35 (1988) 14.

[9] Y.J. Chan, D. Pavlidis, IEEE Trans. Electron. Devices 41 (1994) 637.

[10] D. Biswas, N. Debbar, P. Bhattacharya, M. Razeghi, M. Defour, F. Omnes, Appl. Phys. Lett. 56 (1990) 833.

[11] J.M. Olson, R.K. Ahrenkiel, D.J. Dunlavy, B. Keyes, A.E. Kibbler, Appl. Phys. Lett. 55 (1990) 1208.

[12] J.R. Lothian, J.M. Kuo, F. Ren, S.J. Pearton, J. Electron. Mat. 21 (1992) 441

[13] C.D. Tsai, H.P. Shio, C.T. Lee, Y.K. Tu, IEEE Photon. Technol. Lett. 9 (1997) 660

[14] S. O'Brien, J.R. Shealy, D.P. Bour, Appl. Phys. Lett. 53 (1988) 1859.

[15] V. Swaminathan, A.T. Macrander, Materials Aspects of GaAs and InP Based Structures, Prentice-Hall, Englewood Cliffs, NJ, 1991, p. 19.

[16] K. Iga, H. Uenohara, F. Koyama, Electron. Lett. 22 (1986) 1008.

[17] B. Jonsson, S.T. Eng, IEEE J. Quantum Electron. 26 (1990) 2025.

[18] T. Takagi, F. Koyama, K. Iga, IEEE J. Quantum Electron. 27 (1991) 1511. 